\title{
On the modeling of sound propagation over multi-impedance discontinuities using a semiempirical diffraction formulation
}

\author{
Yiu Wai Lam and Mohammad Reza Monazzam \\ Acoustics Research Centre, School of Computing, Science and Engineering, University of Salford, \\ Salford M5 4WT, United Kingdom
}

(Received 11 October 2005; revised 30 May 2006; accepted 31 May 2006)

\begin{abstract}
Several approximate extensions of the semi-empirical De Jong model [De Jong et al., J. Sound and Vib. 86, 23-46 (1983)] are considered for the prediction of sound propagation over multiple impedance discontinuities. A limitation in the original formulation of the De Jong model is highlighted and a modified form that overcomes this limitation is derived based on reciprocity. This leads to the development of a model for multiple impedance discontinuities that can be used for the investigation of sound-wave propagation above a mixed, striped soft ground that is created by either porous absorbent strips, embedded grooves, or wells with different depths. The accuracy of the model is validated against the boundary element method. It is then used to evaluate the importance of the imaginary part of the admittance of the ground strips on sound attenuation along welled surface. It is shown that the attenuation performance of a surface with multiple impedance discontinuities is high when the imaginary part of the average admittance is large and negative (with an $-i \omega t$ convention), but the magnitudes of the attenuation peaks are also substantially affected by diffraction from the impedance discontinuities. (C) 2006 Acoustical Society of America.
\end{abstract}

[DOI: 10.1121/1.2216905]

PACS number(s): 43.28.En, 43.50.Vt, 43.28.Fp [DKW]

Pages: 686-698

\section{INTRODUCTION}

Sound-wave propagation above a surface with multiple impedance discontinuities has been a subject of continuing interest, particularly due to the possibility of enhancing ground attenuation through diffraction at the discontinuities. A practical application of this problem is in the prediction of traffic noise, for example, which is usually produced above hard ground (e.g., asphalt or concrete), and crosses a discontinuity as it propagates over to a softer ground (e.g., grass or snow). Another example of this problem is in the study of wave propagation over a series of ribs or grooves, which can provide extra attenuation of environmental noise. van Der Heijden and Marten ${ }^{1}$ measured the sound attenuation by a set of parallel wells on the ground, and van Tol and Holties ${ }^{2}$ investigated low, close to track barriers and absorptive layers on a slab track. A further possibility of using such an impedance surface is in novel reactive barrier designs. Fujiwara et $a l .^{3}$ predicted the performance of a series of wells on the top surface of a T-shaped barrier. Recently, the authors showed that adding a Schroeder-type diffuser surface on top of a T-shaped barrier can provide substantial improvement on the barrier's insertion loss. ${ }^{4}$

It should also be noted that the ability of an impedance discontinuity to generate surface waves can also be used to enhance instead of attenuate sound propagation. Zhu et al. ${ }^{5}$ have demonstrated such a possibility by means of an impedance strip with finite width. However, the focus of this paper is attenuation rather than amplification of sound propagating along impedance surfaces. In particular, the possibility of using impedance discontinuities to enhance attenuation is considered.
Many different methods of solution for the case of a two-impedance, single-discontinuity bounsdary have been presented in the literature. Enflo and Enflo ${ }^{6}$ derived a solution in the form of a triple integral. Simpler asymptotic approximations can be obtained when the source and receiver are far from the impedance discontinuity and are both on the ground, and when the distance from the source to the discontinuity is much less than that from the source to the receiver. Rasmussen ${ }^{7}$ also provides an approximate solution for the propagation over an impedance discontinuity. Zhu et al. ${ }^{5}$ showed that this approximation has good agreement with a boundary element method prediction over a simple impedance jump that generates a surface wave.

Unfortunately, the above methods for a singleimpedance discontinuity are not easily amendable to the more complex case of multiple impedance discontinuities that are not necessarily far from each other. Numerical methods of calculations such as parabolic equation ${ }^{8}$ and boundary integral equation ${ }^{9}$ can provide accurate prediction, but they are generally expensive in terms of computation resources and do not always give an insight into the physical mechanisms of the sound attenuation.

The method presented by De Jong et al. ${ }^{10}$ and Koers ${ }^{11}$ can be considered as a different class of solution that uses semiempirical modifications of analytical expressions for diffraction by a rigid half-plane, which is used to represent an admittance step from a hard surface to air. This method has been shown to produce good predictions under a variety of conditions, although Daigle et al. ${ }^{12}$ and Hothersall et al. ${ }^{13}$ found that it has serious limitations in certain geometries involving near-grazing angles and at low frequencies. It was suggested that more accurate results can be obtained at greater source and receiver heights and shorter source to re- 
ceiver distances. Hothersall et al. ${ }^{13}$ also presented an extension of the De Jong model to the case of one impedance strip (two impedance discontinuities) and found good agreement with predictions by the numerical boundary element method. However, as pointed out by Boulanger et al. ${ }^{14}$ their extension has an inconsistency with the original De Jong model. Boulanger et al. ${ }^{14}$ examined the De Jong method at short distances and close to the ground. Very good agreement with measurements was reported for a single discontinuity. However, their extension of the method, which is similar to that given by Bassiouni et al. ${ }^{15}$ and is corrected for the inconsistency noted in the equation of Hothersall et al., ${ }^{13}$ to a surface with periodic multiple impedance strips was found to produce clear discrepancies when compared with measurements.

Nyberg ${ }^{16}$ introduced a solution of the Helmholtz equation for a mixed ground using a Fourier transform technique. He showed that this problem under certain conditions may be estimated by using area-averaged admittance. Although it seems to give a good approximation for a periodic mixed impedance ground in a limited range of frequency, it cannot distinguish the situations where one strip or another is at the specular reflection point for a given percentage of hard surfaces.

The purpose of this paper is to examine the attenuation of sound propagation over a ground with multiple impedance discontinuities that are created by a series of rigid wells with different depths. The width of the wells in the direction of the propagation is generally smaller or comparable to the acoustic wavelength such that the discontinuities cannot be considered to be far from each other. The impedances of these wells can have large variations in values due to well resonances. We wish to derive an approximate analytical solution for this problem to provide some insight into the attenuation mechanism that will allow us to obtain guidelines for design optimization. Of the methods identified from the literature, the approximation method by De Jong is chosen as the basis for further development. First, we examine the De Jong model and explain the apparent inconsistence in the extension ${ }^{13}$ for one impedance strip and the failure of the existing extension ${ }^{14,15}$ for multiple strips. A new extension of the method for multi-impedance ground will then be introduced, and the accuracy of the new model on grounds with single and multiabsorbent strips will be examined. The numerical boundary element method (BEM), which has been shown to be very accurate and reliable in previous studies, ${ }^{9,14}$ will be used as a basis for comparisons and validation. Finally, the model will be applied to multiwelled surfaces with various depth sequences to examine the factors
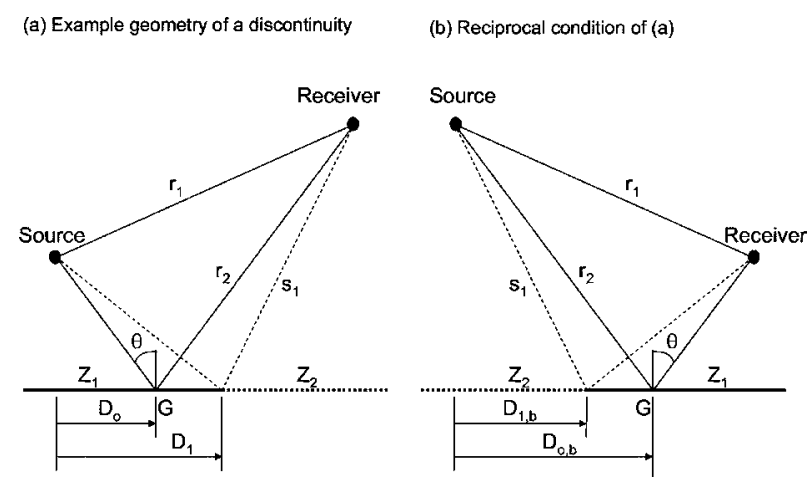

FIG. 1. Definition of the different paths for a single impedance discontinuity. $G$ is the specular reflection point. (a) is an example with the specular reflection point on the source side of the discontinuity and (b) is the reciprocal case of (a).

that influence the excess attenuation, with the view of understanding how such surfaces can affect the performance of a T-shaped barrier.

\section{DIFFRACTION MODELS FOR WAVE PROPAGATION OVER MIXED IMPEDANCE GROUND}

\section{A. Original De Jong model}

De Jong et al. ${ }^{10}$ introduced a solution by comparing the case of an admittance step at a transition between a hard to a soft ground with the case of a semi-infinite screen. It was suggested that the solution for the diffraction by the latter can be used to represent the diffraction by an admittance step from an acoustically hard surface to the atmosphere. Using $e^{-i \omega t}$ to represent the time dependence, the De Jong equation for the relative pressure above a plane containing a singleimpedance discontinuity can be written as

$$
\begin{aligned}
\frac{p}{p_{1}}=1 & +\frac{r_{1}}{r_{2}} Q_{G} e^{i k\left(r_{2}-r_{1}\right)}+\left(Q_{2}-Q_{1}\right) e^{-i \pi / 4} \frac{1}{\sqrt{\pi}} \frac{r_{1}}{s_{1}} \\
& \times\left[F_{2}\left(\sqrt{k\left(s_{1}-r_{1}\right)}\right) \pm F_{2}\left(\sqrt{k\left(s_{1}-r_{2}\right)}\right) e^{i k\left(r_{2}-r_{1}\right)}\right] .
\end{aligned}
$$

The different path lengths including $r_{1}, r_{2}$, and $s_{1}$ are shown in Fig. 1. The total pressure at the receiver point is $p$, and $p_{1}$ is the free-field pressure. $Q_{1}$ and $Q_{2}$ are the spherical wave reflection coefficients calculated for an infinite ground of impedance $Z_{1}$ and $Z_{2}$, respectively, and are calculated at the specular reflection point $G$. Table I summarizes the choices of $Q_{G}$ and the \pm sign inside the right-hand-side square bracket in Eq. (1).

TABLE I. Choice of $Q_{G}$ and $+/-$ sign inside the right-hand side square bracket in the original De Jong model, Eq. (1).

\begin{tabular}{ccc}
\hline \hline & $Z_{1}$ on source side & $Z_{2}$ on source side \\
[e.g., Fig. 1(a) & $Q_{G}=Q_{1}$ & [e.g., Fig. 1(b)] \\
Specular reflection point & $Q_{G}=Q_{1}$ \\
$G$ on impedance $Z_{1}$ & + sign inside the square bracket & - sign inside the square bracket \\
& $\left(Q_{2}-Q_{1}\right)$ in front of square bracket & $\left(Q_{1}-Q_{2}\right)$ in front of square bracket \\
Specular reflection point & $Q_{G}=Q_{2}$ & $Q_{G}=Q_{2}$ \\
$G$ on impedance $Z_{2}$ & - sign inside the square bracket & + sign inside the square bracket \\
& $\left(Q_{2}-Q_{1}\right)$ in front of square bracket & $\left(Q_{1}-Q_{2}\right)$ in front of square bracket \\
\hline \hline
\end{tabular}


The third term on the right-hand side of the equation can be seen as the contribution from the diffraction by the impedance discontinuity, and is equal to a correction term $\left(Q_{2}-Q_{1}\right)$ times the approximate diffracted field from a hard half-plane. The term $\left(Q_{2}-Q_{1}\right)$ corrects for the surfaces having finite impedance $Z_{1}$ and $Z_{2}$. It is empirically constructed by physical arguments to give the correct prediction when $Q_{1}=1$ (acoustically hard) and $Q_{2}=0$ (air), and when $Z_{1}$ approaches $Z_{2}$. Because of the empirical nature of this correction, its limitations are not mathematically explicit. Daigle et $a l{ }^{12}$ conducted a thorough study on the behavior of the De Jong model. They found that the model has difficulties dealing with situations when the grazing angles are small, but they were not able to formulate firm criteria for the use of the model.

Of the diffraction terms inside the square bracket in Eq. (1), $F_{2}(x)$ is the integral

$$
F_{2}(x)=\int_{x}^{\infty} e^{\left(i w^{2}\right)} d w
$$

The first $F_{2}$ term inside the square bracket can be considered as the diffraction contribution associated with the direct source, while the second $F_{2}$ term is that associated with the image source. The sign change for the second term is therefore understandable as the reflected path $r_{2}$ moves from the source side to the receiver side of the discontinuity. Note that the diffraction terms used here are derived with an assumption that $k s_{1} \gg 1$. Daigle et al. ${ }^{12}$ found that the differences between the use of this approximation and an exact formulation of the diffracted field in the De Jong model are not significant in the cases that they studied. The observed errors of the model at small grazing angles are not caused by the approximation of the hard half-plane diffracted field, but rather by the empirical correction for the finite impedances.

The spherical reflection coefficient can be calculated by the Weyl-van der Pol approximation, ${ }^{17}$

$$
Q=R_{P}+\left(1-R_{P}\right) F(z)
$$

where $R_{p}$ is the plane-wave reflection coefficient,

$$
R_{p}=\frac{\cos \theta-\beta}{\cos \theta+\beta} .
$$

In this paper the normalized surface admittance $\beta$ for absorptive fibrous material is calculated by the empirical equations of Delany and Bazely. ${ }^{18}$ For narrow wells, the model described by Eq. (8) of $\mathrm{Wu}$ et al. ${ }^{19}$ is used to compute $\beta$. Briefly, the model assumes plane-wave propagation in the well but adjusted to incorporate the effects of thermal wave and shear wave in the boundary layers. Full details can be found in Sec. I A of Ref. 19.

The argument $z$ in the function $F(z)$ is called the numerical distance. To help the discussion in later sections, the following equations, which are well documented in the literature, ${ }^{20-22}$ are given:

$$
z=+\frac{1+i}{2} \sqrt{k r_{2}}(\beta+\cos \theta)
$$

The function $F(z)$, which describes the interaction between the spherical wavefront with the impedance boundary, is approximated by

$$
F(z)=1+i z \sqrt{\pi} \operatorname{cerfe}(z),
$$

where $\operatorname{cerfe}(z)$ is the scaled complemented error function. In the range of $|\beta|^{2} k r_{2} \gg 1$, the absolute value of the numerical distance is much bigger than unity. The error function complement can then be approximated by an asymptotic expansion, and the boundary factor $F(z)$ for large $|z|$ may be written as

$$
F(z)=2 i \sqrt{\pi} z H[-\operatorname{Im}(z)] e^{-z^{2}}-\left\{\frac{1}{2 z^{2}}+\frac{3}{4 z^{4}}+\cdots\right\},
$$

where $H$ is the Heaviside step function-it is unity when its argument is positive, and zero when its argument is negative.

The excess attenuation can now be calculated as

$$
\mathrm{EA}=-20 \log \left(\frac{p}{p_{1}}\right) \mathrm{dB} \text {. }
$$

\section{B. Modified De Jong model}

The original De Jong model is derived for the case of an impedance step from a harder ground to a softer ground $\left(Z_{1}\right\rangle Z_{2}$ in Fig. 1). The model has been shown by De Jong et al. and others ${ }^{10,13,14}$ to have good accuracy under a variety of conditions for a single-impedance jump from hard to soft ground, which satisfy De Jong's assumption of hard to soft transition in his derivation of the model. Unfortunately, this assumption has been overlooked in other studies and caused some confusion when the model is extended to cover multiple impedance discontinuities. The implication of this assumption of hard to soft transition can be clearly seen by considering Eq. (1) under reciprocal conditions. An example is shown in Fig. 1, where Fig. 1(b) is the reciprocal case of Fig. 1(a). Applying the original De Jong model, i.e., Eq. (1), to Fig. 1(a), gives the total pressure $p_{a}$ as

$$
\begin{aligned}
\frac{p_{a}}{p_{1}}=1 & +\frac{r_{1}}{r_{2}} Q_{1} e^{i k\left(r_{2}-r_{1}\right)}+\left(Q_{2}-Q_{1}\right) e^{-i \pi / 4} \frac{1}{\sqrt{\pi}} \frac{r_{1}}{s_{1}} \\
& \times\left[F_{2}\left(\sqrt{k\left(s_{1}-r_{1}\right)}\right)+F_{2}\left(\sqrt{k\left(s_{1}-r_{2}\right)}\right) e^{i k\left(r_{2}-r_{1}\right)}\right] .
\end{aligned}
$$

On the other hand, applying the model to the reciprocal case of Fig. 1(b) gives the total pressure $p_{b}$ as

$$
\begin{aligned}
\frac{p_{b}}{p_{1}}= & 1+\frac{r_{1}}{r_{2}} Q_{1} e^{i k\left(r_{2}-r_{1}\right)}+\left(Q_{2}-Q_{1}\right) e^{-i \pi / 4} \frac{1}{\sqrt{\pi}} \frac{r_{1}}{s_{1}} \\
& \times\left[-F_{2}\left(\sqrt{k\left(s_{1}-r_{1}\right)}\right)+F_{2}\left(\sqrt{k\left(s_{1}-r_{2}\right)}\right) e^{i k\left(r_{2}-r_{1}\right)}\right] .
\end{aligned}
$$

Note that in Eq. (10) we have already adjusted for the reversal of the $\left(Q_{1}-Q_{2}\right)$ term and the change of sign of the second $F_{2}$ term inside the square bracket for the reciprocal case. It can be clearly seen that Eq. (10) differs from Eq. (9) by the negative sign in front of the first $F_{2}$ term inside the square 
bracket. Consequently $p_{a} \neq p_{b}$, which violates the reciprocity condition. Due to this difference the original model, i.e., Eq. (1), does not satisfy acoustic reciprocity and is therefore invalid for a soft to hard transition.

In order for the model to satisfy the reciprocity condition, we can impose a further condition on the sign of the first $F_{2}$ term. By comparing Eqs. (9) and (10), and knowing that Eq. (1) works for hard to soft transition, we modified the De Jong model into the following equation:

$$
\begin{aligned}
\frac{p}{p_{1}}= & 1+\frac{r_{1}}{r_{2}} Q_{G} e^{i k\left(r_{2}-r_{1}\right)}+\left(Q_{2}-Q_{1}\right) e^{-i \pi / 4} \frac{1}{\sqrt{\pi}} \frac{r_{1}}{s_{1}} \\
& \times\left[\mu F_{2}\left(\sqrt{k\left(s_{1}-r_{1}\right)}\right)+\gamma F_{2}\left(\sqrt{k\left(s_{1}-r_{2}\right)}\right) e^{i k\left(r_{2}-r_{1}\right)}\right],
\end{aligned}
$$

where, as before, $\gamma=1$ for $D_{o}<D_{1}$ and $=-1$ for $D_{o}>D_{1}$. The new parameter $\mu$ is introduced to account for the reciprocity requirement such that $\mu=1$ when the admittance $\beta_{2}>\beta_{1}$, and $=-1$ for $\beta_{2}<\beta_{1}$. Equation (11) will be referred to as the modified De Jong model.

\section{Extension to multiple impedance discontinuities}

Due to the assumption of hard to soft transition in the original De Jong model, any extension of this model to multiple impedance discontinuities that involves transitions to a harder ground is bound to be in error. Bassiouni et al. ${ }^{15}$ extended the De Jong method for the sound propagation over a ground surface containing any finite number of impedance discontinuities. Their equation is a straightforward application of the original De Jong model to each of the impedance discontinuities in turn. As such, it will not work if any of the consecutive impedance changes is from a softer to a harder ground. Unfortunately, Bassiouni et al. ${ }^{15}$ does not contain any data to support its claim of the model's accuracy.

Boulanger et al. ${ }^{14}$ used the same approach to extend the original De Jong model to calculate sound propagation over a ground with periodic impedance changes. Equation (18) of Boulanger et al. ${ }^{14}$ is essentially the same as Bassiouni's equation, but simplified to periodic changes between two impedances. This periodic impedance changes clearly violate the assumption of only hard to soft transition in the original De Jong model. Therefore, it is not surprising that the extended model shows clear discrepancies when compared with measured data (see Fig. 12 of Boulanger et al. ${ }^{14}$ ).

In another study, Hothersall et al. ${ }^{13}$ presented an extension of the De Jong model to one strip of impedance change, i.e., two impedance discontinuities. In their description of the De Jong model, they have in fact correctly stated that the choice of $Z_{2}$ and $Z_{1}$ in Eq. (1) should be such that $Z_{1}$ is the more rigid of the two. In their extension of the De Jong model to two discontinuities, there is a sign change in the Fresnel integral that corresponds to the $F_{2}\left(\sqrt{k\left(s_{1}-r_{1}\right)}\right)$ term [see Eqs. (10)-(12) in Hothersall et al. ${ }^{13}$ ] that accounts for the diffraction from the second discontinuity (transition from softer to harder impedance). Unfortunately, they did not give any description or justification for the change. Indeed, they suggested that their equations follow Bassiouni's approach ${ }^{15}$ and did not point out the differences. Consequently, there is confusion in later literature ${ }^{14}$ that claims that their equations

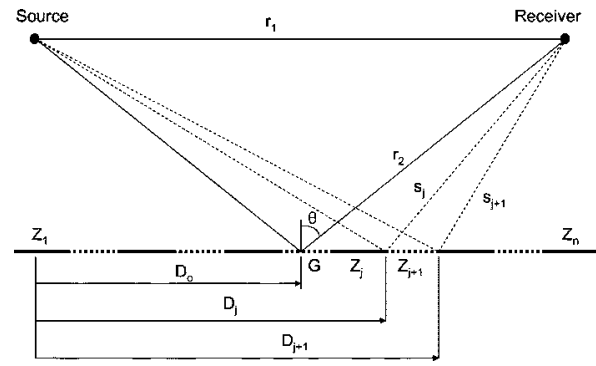

FIG. 2. Definition of the different paths for multiple impedance discontinuities. $G$ is the specular reflection point.

were inconsistent with De Jong's model, and it was suggested $^{14}$ that $a-1$ should be multiplied to the Fresnel integral in question. It is now clear that that suggestion is incorrect and should not be carried out.

\section{The new Multi-impedance Discontinuities Model (nMID)}

The above observations clearly show that the original De Jong model [Eq. (1)] cannot be extended to cases with multiple impedance discontinuities without incorporating the modifications suggested here in Eq. (11) to deal with the case of an impedance transition from softer to harder ground. Based on reciprocity requirement, it has been shown earlier that a sign change in the diffraction term associated with the direct path is necessary to account for the soft to hard transition. Based on this modification, we can now extend the modified De Jong model to multiple impedance ground by applying Eq. (11) to each of the discontinuities in turn. For a ground with $n$ impedance sections, and hence $(n-1)$ impedance discontinuities, the total pressure $p$ is approximately given by

$$
\begin{aligned}
\frac{p}{p_{1}}= & 1+\frac{r_{1}}{r_{2}} Q_{G} e^{i k\left(r_{2}-r_{1}\right)}+\sum_{j=1}^{n-1}\left(Q_{j+1}-Q_{j}\right) \frac{e^{-i \pi / 4}}{\sqrt{\pi}} \frac{r_{1}}{s_{j}} \\
& \times\left[\mu_{j} F_{2}\left(\sqrt{k\left(s_{j}-r_{1}\right)}\right)+\gamma_{j} F_{2}\left(\sqrt{k\left(s_{j}-r_{2}\right)}\right) e^{i k\left(r_{2}-r_{1}\right)}\right],
\end{aligned}
$$

where $\gamma_{j}=1$ for $D_{o}<D_{j}$ and $=-1$ for $D_{o}>D_{j}$, and $\mu_{j}=1$ when the admittance $\beta_{j+1}>\beta_{j}$ and $=-1$ for $\beta_{j+1}<\beta_{j}$. The geometry and the definition of the paths and symbols are as shown in Fig. 2.

Obviously this straightforward application of the singlediscontinuity diffraction formula, Eq. (11), to multiple discontinuities will inherit the limitation of the base formula, i.e., that the accuracy decreases with larger reflection angles (nearer grazing). Furthermore, such a simple combination does not consider possible interactions between the discontinuities, and is therefore expected to work best when the discontinuities are far apart relative to the acoustic wavelength. Note that when there is only one impedance strip, i.e., $n=3$ with $Z_{3}=Z_{1}$, Eq. (12) reduces to the same Eqs. (10)-(12) of Hothersall et al. ${ }^{13}$

Also, for a ground with a periodic arrangement of identical strips of impedance $Z_{2}$ embedded in a ground of impedance $Z_{1}$, such as the case considered in Boulanger et al. ${ }^{14}$ the equation can be rewritten in terms of the diffraction terms from the two edges of each strip as 


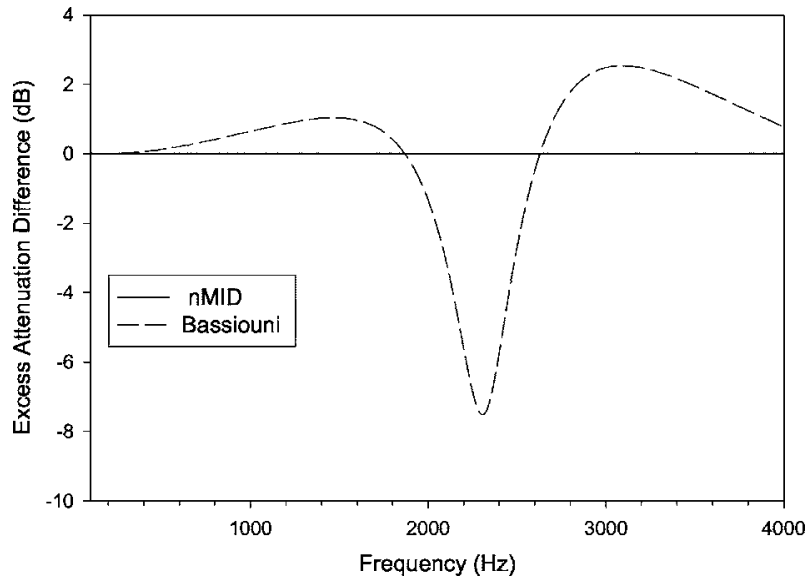

FIG. 3. Error in applying the original De Jong model to reciprocal cases of propagation over a hard ground with a $0.12-\mathrm{m}$ absorbent strip placed at the midpoint between source and receiver. Distance from source to receiver is $1 \mathrm{~m}$.

$$
\begin{aligned}
\frac{p}{p_{1}}= & 1+\frac{r_{1}}{r_{2}} Q_{G} e^{i k\left(r_{2}-r_{1}\right)}+\left(Q_{2}-Q_{1}\right) \frac{e^{-i \pi / 4}}{\sqrt{\pi}} \\
& \times \sum_{k=1}^{m}\left[\frac { r _ { 1 } } { s _ { 2 k - 1 } } \left\{F_{2}\left(\sqrt{k\left(s_{2 k-1}-r_{1}\right)}\right)+\gamma_{2 k-1}\right.\right. \\
& \left.\times F_{2}\left(\sqrt{k\left(s_{2 k-1}-r_{2}\right)}\right) e^{i k\left(r_{2}-r_{1}\right)}\right\}+\frac{r_{1}}{s_{2 k}} \\
& \left.\times\left\{F_{2}\left(\sqrt{k\left(s_{2 k}-r_{1}\right)}\right)-\gamma_{2 k} F_{2}\left(\sqrt{k\left(s_{2 k}-r_{2}\right)}\right) e^{i k\left(r_{2}-r_{1}\right)}\right\}\right],
\end{aligned}
$$

where $m$ is the total number of strips.

\section{E. The significance of the modification}

In Sec. II B we have shown mathematically that the original De Jong model does not satisfy reciprocity requirement. The consequence of this in application to a simple case of a single-impedance strip (two impedance discontinuities) is shown in Fig. 3. The impedance strip is placed perpendicular to the source to receiver direction and has a width of $12 \mathrm{~cm}$. This width is much narrower, compared with acoustic wavelength, than those used in a previous study. ${ }^{13}$ The reason for choosing such a narrow width is that our eventual purpose is to use the model to investigate the excess attenuation created by embedded wells that have widths that are less than the acoustic wavelength. In Fig. 3 the strip has a porous-type flow resistivity $\left(200000 \mathrm{~N} \mathrm{~s} \mathrm{~m}^{-4}\right)$ that is typical of grassland. The source to receiver distance is $1 \mathrm{~m}$, and the strip is placed on a rigid ground midway between the source and the receiver. Calculations are first made for a source height of $0.1 \mathrm{~m}$ and a receiver height of $0.4 \mathrm{~m}$, and then for the corresponding reciprocal situation. The differences in the excess attenuation between the two cases are shown in Fig. 3. The result from the extension by Bassiouni et al., which is based on the original De Jong model, is labeled as "Bassiouni."

Because the two cases are the reciprocal of each other, we expect the difference between the calculated excess at-

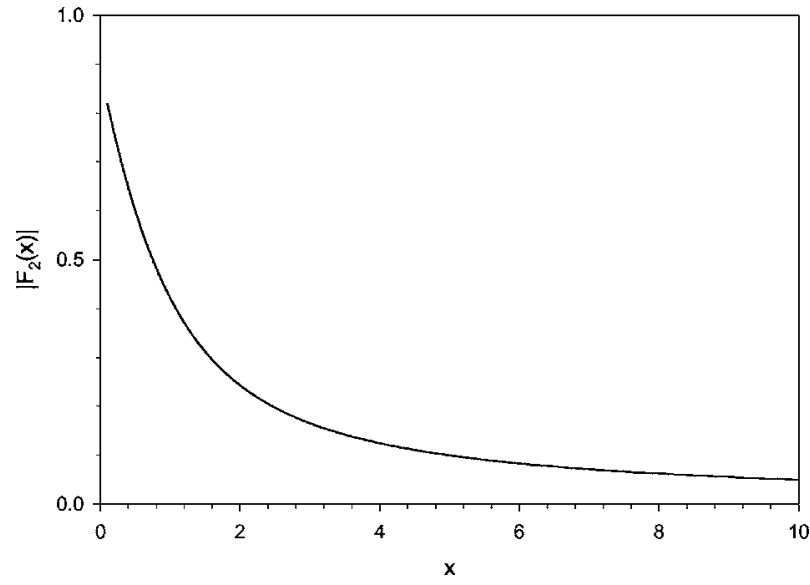

FIG. 4. The absolute value of the Fresnel integral $F_{2}(x)$.

tenuation to be exactly zero. This is indeed the case with the nMID model. On the other hand the extension (Bassiouni) based on the unmodified De Jong model produces differences of up to $8 \mathrm{~dB}$, proving that it does not satisfy the reciprocity requirement.

Another way to look at the significance of the modification is to examine the differences between the equations representing the two models. The only difference between the modified model, Eq. (11), and the original De Jong model, Eq. (1), is the reversal of the sign of the $F_{2}\left(\sqrt{k\left(s_{1}-r_{1}\right)}\right)$ term when the transition is from a softer impedance to a harder impedance, i.e., the introduction of the parameter $\mu$ in Eq. (11). Hence, the significance of the modification can be seen by examining the behavior of this $F_{2}$ term. Figure 4 shows the absolute value of this term as a function of its argument $x$, which is given by $\sqrt{k\left(s_{1}-r_{1}\right)}$. As can be seen from the figure, the value of $F_{2}$ increases as its argument gets smaller. Therefore, it is expected that the effect of the modification, or in other words the error in any extensions to multiple impedance strips that are based on the original formulation, will be greater when $\sqrt{k\left(s_{1}-r_{1}\right)}$ is smaller. This occurs at lower frequencies and, from the geometry of Figs. 1 and 2, when the source and receiver heights are low and the propagation is close to grazing. Generally the nMID model provides more significant improvements over the incorrect model at lower frequencies and at smaller grazing angles. Note that this only applies to soft to hard impedance transitions, but in the case of multiple impedance strips such transitions will inevitably occur. To demonstrate this, Fig. 5 compares the result of predictions using the nMID model and Bassiouni's equation on the single-impedance strip configuration of Fig. 10(a) of Hothersall et al. ${ }^{13}$ The source and receiver heights are both at $4 \mathrm{~m}$. The distance between them is $20 \mathrm{~m}$. The strip width is $5 \mathrm{~m}$. The position of the strip is changed from below the source to below the receiver. The excess attenuation is plotted against the horizontal distance from the source to the center of the strip in Fig. 5 for the frequency of $500 \mathrm{~Hz}$. The flow resistivity of the strip is taken to be $200000 \mathrm{~N} \mathrm{~s} \mathrm{~m}^{-4}$ as in Hothersall et al., ${ }^{13}$ but the depth of the absorbent is taken to be $0.032 \mathrm{~m}$. The source and receiver geometry is symmetrical. Therefore, we expect the results to be symmetrical about the center point (when the distance from the source to the center of the strip is $10 \mathrm{~m}$ ). In 


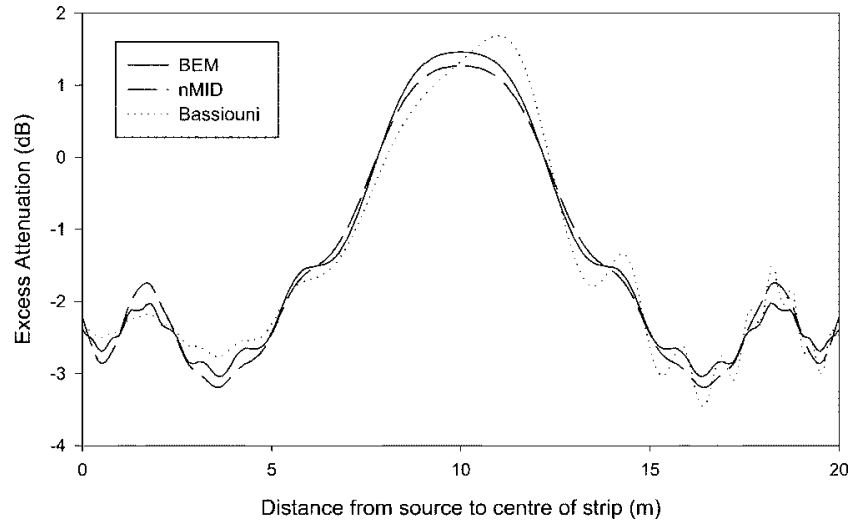

FIG. 5. Comparison between calculations of propagation over an absorbent strip of width $5 \mathrm{~m}$. Source and receiver heights are both $4 \mathrm{~m}$. Distance from source to receiver is $20 \mathrm{~m}$. The source frequency is $500 \mathrm{~Hz}$.

this configuration the nMID is the same as Eqs. (10)-(12) of Hothersall et al. ${ }^{13}$ Indeed, the nMID model shows good agreement with BEM that is similar to that found in Hothersall et al. ${ }^{13}$ On the other hand, the incorrect Bassiouni model shows an asymmetric result in Fig. 5 because of the lack of reciprocity in its formulation.

One particular case where extensions based on the original De Jong model will always fail to predict the correct attenuation occurs when the specular reflection point is outside but close to either side of a narrow impedance strip. This can be seen by examining the nMID model for a single impedance strip [i.e., $m=1$ in Eq. (13)], and with the reflection point at the source side of the strip. Under this condition the incorrect extension based on the original De Jong equation (e.g., Bassiouni et al. ${ }^{15}$ and Boulanger et al. ${ }^{14}$ ) is similar to the nMID model but has a -1 sign in front of the third Fresnel integral inside the square bracket [i.e., the $F_{2}\left(\sqrt{k\left(s_{2}-r_{1}\right)}\right)$ term]. If the strip is narrow such that the difference between $s_{1}$ and $s_{2}$ is small, then the $F_{2}$ terms inside the square bracket in this incorrect extension will largely cancel. This will then leave only the first two terms (representing spherical reflection from an infinite ground) in the equation, even though the discontinuities are close to the specular reflection point. On the other hand, the nMID model will have correctly a significant diffraction contribution from the addition of the two $F_{2}$ terms associated with $r_{1}$. The difference is large especially when the difference between the two impedances is large such that $\left(Q_{2}-Q_{1}\right)$ returns a large value. One such example is when the impedance strip is created by a well that has a large swing of extreme impedance values due to resonances in the well at different frequencies. Figure 6 shows the result of such a case. The strip is a narrow well of depth $0.3 \mathrm{~m}$ and a width (in the source to receiver direction) of $0.12 \mathrm{~m}$. It is placed on a rigid ground midway between the source and the receiver. The source height is $0.1 \mathrm{~m}$ and the receiver height is $0.2 \mathrm{~m}$. The source to receiver distance is $1 \mathrm{~m}$. The reflection point is on the source side of the strip. The figure shows clearly the failure of the incorrect extension (labeled "Bassiouni" in the figure) to predict the diffraction effect from the strip. In comparison, the nMID prediction clearly shows strong attenuation patterns created by the embedded well.

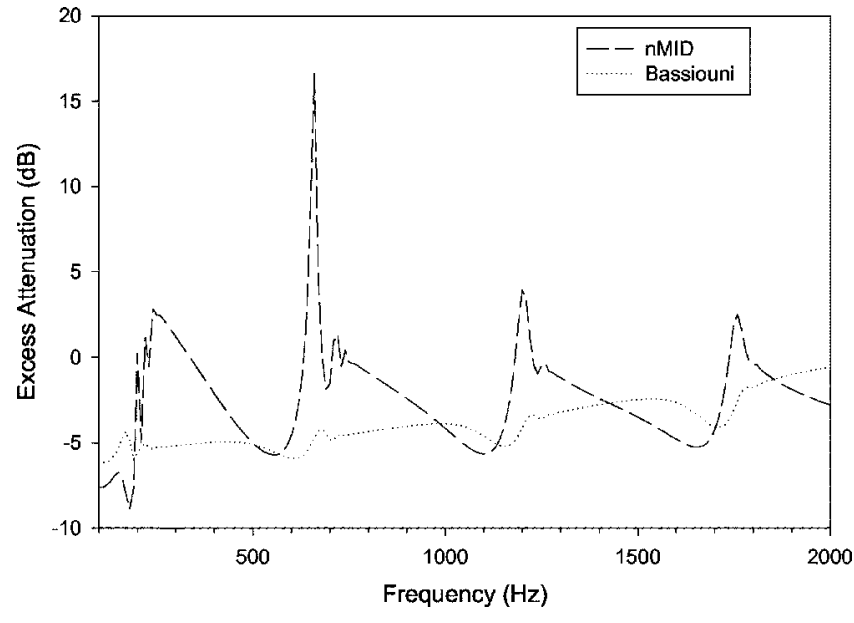

FIG. 6. Predicted attenuation over a rigid ground with a well of width $0.12 \mathrm{~m}$ and depth $0.3 \mathrm{~m}$ located midway between source and receiver. Distance from source to receiver is $10 \mathrm{~m}$ and source and receiver heights are 0.1 and $0.2 \mathrm{~m}$, respectively.

\section{PROPAGATION OVER MIXED POROUS IMPEDANCE SURFACES}

Although there have been previous studies ${ }^{13-15}$ in the literature on the application of De Jong's model to mixed impedance grounds, not all the results are positive due to the incorrect application of the original De Jong model and the confusion over the apparent inconsistence ${ }^{14}$ of Hothersall et al.'s equations ${ }^{13}$ with the De Jong equation. Hothersall et $a l{ }^{13}$ studied single-impedance strip configurations with strips that were 5 to $10 \mathrm{~m}$ wide at frequencies not lower than $500 \mathrm{~Hz}$. Good accuracy was found except at close to grazing propagation. Because of our eventual goal of using the nMID model for wells that are narrower than a wavelength, we wish to conduct further investigations on propagation over narrow impedance strips. In all our tests, the strips are all $0.12 \mathrm{~m}$ wide, and the source to receiver distance is always $1 \mathrm{~m}$. The source and receiver heights are in the range from close to the ground to $0.4 \mathrm{~m}$. In other words the effect of mixed impedance boundaries that are close to each other is considered in situations where source and receiver are close to the surface and separated by a short distance. The short separation of the source and receiver provides a reflection angle $\theta$ that is largely different from grazing (about $60^{\circ}$ to $70^{\circ}$ from the normal of the reflecting surface in most cases) for most of the source and receiver heights of interest. With this arrangement the path lengths $s_{j}$ (see Fig. 2) are of the order of $1 \mathrm{~m}$. The assumption of $k s_{j} \gg 1$ that is inherent to the diffraction approximation used in the nMID model should therefore be satisfied at frequencies above $300 \mathrm{~Hz}$ when $k s_{j}>5$.

The accuracy of the nMID model is first examined on a single absorbent strip, and then on a mixed impedance surfaces in which seven different admittance strips in a rigid ground are used. As in Hothersall et al., ${ }^{13}$ the boundary element method, which has been shown to have good agreement with measured data in previous works on mixed impedance plans, ${ }^{9,14}$ is used to provide data for the validation of 


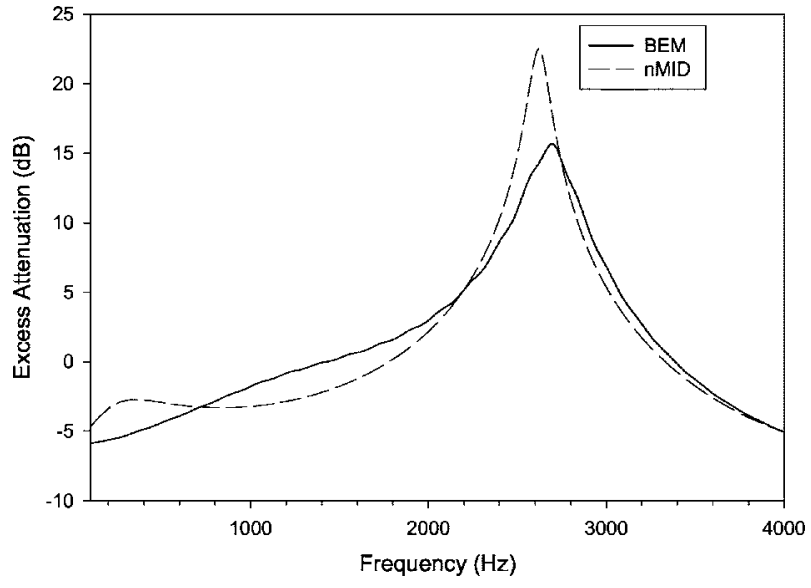

FIG. 7. Propagation over one porous absorbent strip. Source and receiver heights are 0.1 and $0.4 \mathrm{~m}$, respectively, the strip is located at midpoint between source and receiver. The flow resistivity $\sigma=20000 \mathrm{~N} \mathrm{~s} \mathrm{~m}^{-4}$.

the nMID model. Full details of the boundary element method (BEM) used in this investigation can be found in Monazzam and Lam. ${ }^{4}$

\section{A. One absorbent strip}

Figure 7 shows the prediction of the excess attenuation for wave propagation over a narrow strip of porous material (flow resistivity $\sigma=20000 \mathrm{~N} \mathrm{~s} \mathrm{~m}^{-4}$ ). The source and receiver heights are 0.1 and $0.4 \mathrm{~m}$, respectively. It can be seen that the nMID model has good agreement with the BEM prediction except for the attenuation peak at around $2.5 \mathrm{kHz}$. The reflection angle $\theta$ is $63.4^{\circ}$ and should be far enough from grazing for the nMID model to work. On the other hand, the width of the strip, $12 \mathrm{~cm}$, is less than an acoustic wavelength at frequencies below about $2.8 \mathrm{kHz}$. The discrepancy in the attenuation peak is therefore likely to be caused by the interactions between the two discontinuities due to their small separation (less than a wavelength below $2.8 \mathrm{kHz}$ ). In this case the BEM prediction, which takes into account the interactions, has a significantly lower peak magnitude. However, the interactions do not always result in a reduction in peak magnitude, as it can be seen in the example in the next section where the magnitude predicted by the BEM can be smaller than that predicted by the nMID model. Overall though the main features of the attenuation variation with frequency are well predicted. The nMID model appears to provide a reasonably good prediction of the attenuation even when the two impedance discontinuities are closer than a wavelength to each other.

\section{B. Multiabsorbent strips}

A mixed surface with seven absorbent strips placed back to back to each other and located perpendicularly to the direction from source to receiver on a rigid ground is used for this investigation. The predictions by the nMID and by the BEM are shown in Fig. 8. The surface in Fig. 8 has an arbitrary arrangement of absorbent strips. The flow resisitivities of the absorbents, which are stated in the figure, are chosen to be typical of soft to grass-covered grounds. The strips are all given a fixed depth of $0.1 \mathrm{~m}$ for the simulation. (a)

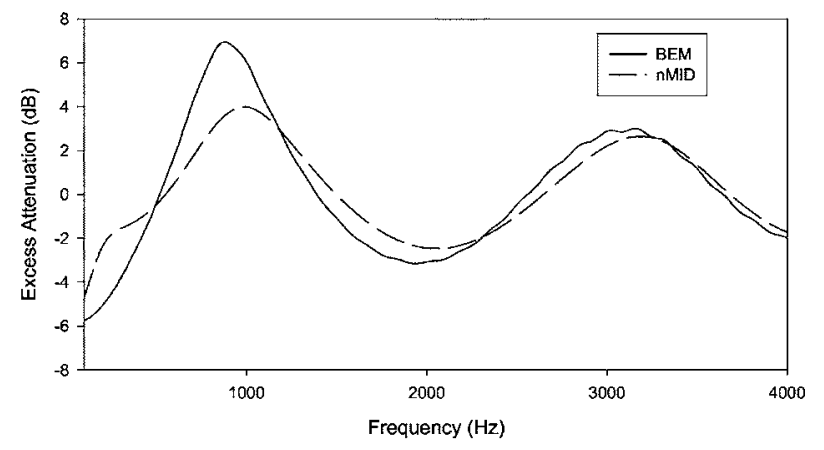

(b)

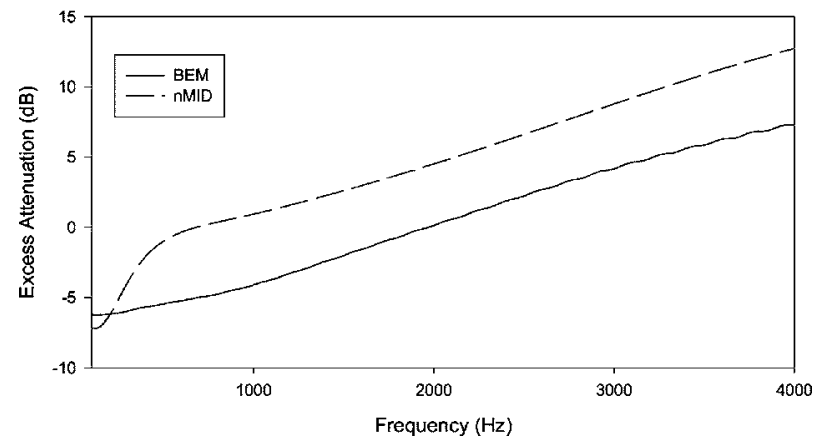

FIG. 8. Wave propagation over a mixed impedance surface containing seven strips placed on a rigid ground midway between the source and receiver. The flow resistivity of the strips from source to receiver are 250000,200000 , $20000,300000,120000,180000$, and $350000 \mathrm{~N} \mathrm{~s} \mathrm{~m}^{-4}$. Source and receiver heights are (a) 0.4 and $0.2 \mathrm{~m}$; (b) 0.01 and $0.01 \mathrm{~m}$, respectively.

Reasonable agreement is seen between the nMID model and the boundary element method in Fig. 8(a) when the source and receiver are high above the surface $(0.4$ and $0.2 \mathrm{~m}$ high, respectively, with a separation of $1 \mathrm{~m}$ ). The closeness of the discontinuities once again created shifts in the frequency and the magnitude of the attenuation peaks that are similar to that seen in Fig. 7, which has similar source and receiver heights. Again, the overall trend of the attenuation is well predicted. As the source and receiver are moved close to the ground (both are $0.01 \mathrm{~m}$ above ground and the reflection angle becomes $\theta=88.9^{\circ}$ ) in Fig. 8(b), a consistent error relative to the BEM prediction is seen over most of the frequency range shown in the figure. This consistent error seems to be a result of the limited accuracy of the De Jong model when the propagation is close to grazing. Apart from the constant shift in magnitude, the nMID still matches the trend of the attenuation increase with frequency that is predicted by the BEM. It seems that the nMID model can provide a good indication of the essential features of the attenuation.

\section{PROPAGATION OVER WELLED SURFACES}

\section{A. Effect of imaginary part of admittance for welled surface}

The use of wells of different depths to create a mixed impedance surface is of particular interest here. This is the base design for the well-known Schroeder-type diffusers. It has been shown by numerical simulation that putting such a 
surface on top of a T-shaped barrier can substantially increase the insert loss of the barrier. ${ }^{4}$ However, modeling such welled surfaces in the analytical multiple impedance discontinuities model presents more difficulties than modeling porous absorbent strips. With a porous strip, the surface admittance (the inverse of impedance) varies smoothly with frequency. The real part, which corresponds to resistive loss, is generally significant. When the strip is created by a well, the admittance at the entrance to the well can vary widely due to resonances. For example, for a rigid well that is narrow enough such that plane wave propagation can be assumed, the value of the normalized input admittance $\beta$ changes from negative infinity to positive infinity when passing through a resonance.
The behavior of the spherical wave boundary factor $F(z)$, Eq. (6), has been studied extensively in the past. ${ }^{23}$ It is known that the approximation is only valid when the value of $\beta$ satisfies certain conditions. Additionally, the attenuation effect represented by the boundary factor $F(z)$ is likely to change considerably due to the extreme variation of the $\beta$ value with frequency for welled surfaces. Hence, it is necessary to examine the effect of $\beta$ on the spherical reflection coefficient for propagation over a boundary with embedded rigid wells.

The boundary factor $F(z)$ can be approximated by Eq. (7) for large values of the numerical distance $|z|$. However, Eq. (7) is only bounded for certain range of the argument of $z$. The suggested substitution by Stinson ${ }^{23}$ for Eq. (7) is

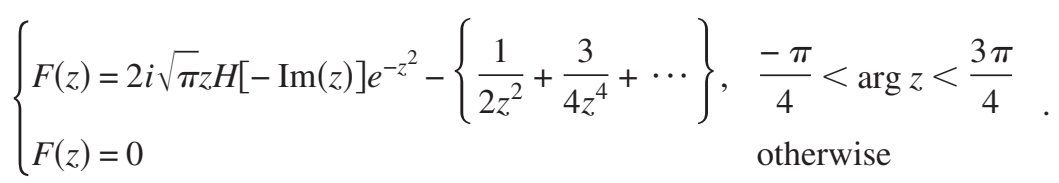

From Eq. (5) the real and imaginary part of the numerical distance $z$ are given by

$$
\begin{aligned}
& \operatorname{real}(z)=\frac{\sqrt{k r_{2}}}{2}(a-b+\cos \theta), \text { and } \\
& \operatorname{imag}(z)=\frac{\sqrt{k r_{2}}}{2}(a+b+\cos \theta), \\
& \arg (z)=\arctan \left(\frac{a+b+\cos \theta}{a-b+\cos \theta}\right),
\end{aligned}
$$

where $a$ and $b$ are the real and imaginary parts of the admittance $\beta$, respectively. The real part of the numerical distance $z$ is negative if the imaginary part of the admittance $\beta$ is positive and bigger than the sum of the cosine of the reflection angle and the real part of the admittance. At the entrance to a rigid well, the real part of the admittance is positive but very small $(\approx 0)$, but the imaginary part is very large, particularly around resonant frequencies. When the reflection angle $\theta$ is large and therefore $\cos \theta$ is small, $\arg (z)$ is determined mainly by $b$, the imaginary part of $\beta$. From Eq. (15), a large and positive imaginary part of the admittance $\beta$ (i.e., masslike reactance) will result in the real part of numerical distance $z$ being negative and the imaginary part of $z$ being positive. When the imaginary part of $\beta$ is large and negative (i.e., springlike reactance), the real part of the numerical distance $z$ is positive and the imaginary part of $z$ is negative. In both cases, since the real part of $\beta$ is positive and small for a rigid well, we have $|a| \ll|b|$ and the value of $\arg (z)$ is always within the valid range $(-\pi / 4<\arg z<3 \pi / 4)$. Therefore, Eq. (7) is valid for the welled surface. However, $\arg (z)$ will be close to the limit of $3 \pi / 4$ when the imaginary part of $\beta$ is large and posi- tive, and close to $-\pi / 4$ when the imaginary part of $\beta$ is large and negative.

A closer look at the equations for the spherical reflection coefficient can also reveal the behavior of the apparent sound attenuation over a welled surface at resonant frequencies where large values of admittance $\beta$ occur. From Eq. (4) it can be seen that the plane-wave reflection coefficient approaches -1 when $\beta$ is much larger than $\cos \theta$, where $\theta$ is the reflection angle from the surface normal. The numerical distance $z$ is large when $\beta$ is large. For large values of $z$, the boundary factor $F(z)$, from Eq. (7), will approach zero. Hence, the spherical reflection coefficient, Eq. (3), will approach -1 when $\beta$ is large at resonance. For a surface with a uniform admittance and without discontinuities, the sound propagation is given by Eq. (1) without the diffraction terms [the third term on the right-hand side of Eq. (1)], and with $Q_{G}$ calculated from the admittance of the well. When $Q_{G}$ approaches -1 , the reflected pressure largely cancels the incident pressure to give large ground attenuation. The amplitude factor $\left(r_{1} / r_{2}\right)$ and the phase factor $e^{i k\left(r_{2}-r_{1}\right)}$ will modify the exact frequency at which the attenuation peak occurs, but it will be close to the resonant frequencies of the well, especially at lower frequencies. This is what one would expect since physically the sound energy will be trapped by the resonating well. Therefore, we can expect the imaginary part of the admittance $\beta$ of rigid wells to have a significant correlation with the attenuation of sound propagation over a surface embedded with such rigid wells.

When there is an impedance discontinuity nearby, the reflected pressure is modified by the diffraction terms in Eq. (1), even if the reflection point is still on the surface of the well. To see how the discontinuity modifies the attenuation, we can make further approximations to simplify Eq. (1). Since the integral $F_{2}$ decays rapidly with its argument (Fig. 
4), one may ignore the first $F_{2}$ term that involves the bigger argument $\sqrt{k\left(s_{1}-r_{1}\right)}$ when the source and receiver are not close to the ground, and write

$$
\frac{p}{p_{1}} \approx 1+\frac{r_{1}}{r_{2}} e^{i k\left(r_{2}-r_{1}\right)} \Gamma,
$$

where

$$
\Gamma=\left[Q_{2}-\left(Q_{2}-Q_{1}\right) \frac{(1-i)}{\sqrt{2 \pi}} F_{2}(x)\right], \text { with } x=\sqrt{k\left(s_{1}-r_{2}\right)} .
$$

If the ground reflection point is close to the discontinuity, then $x$ is small and a crude approximation of the $F_{2}$ integral based on a simple step approximation of the integration is

$$
F_{2}(x) \approx\left(\sqrt{\frac{\pi}{8}-x}\right)+i \sqrt{\frac{\pi}{8}},
$$

and $\Gamma$ is then

$$
\Gamma=\left[Q_{2}-\left(Q_{2}-Q_{1}\right)\left\{\left(\frac{1}{2}-\frac{x}{\sqrt{2 \pi}}\right)+i \frac{x}{\sqrt{2 \pi}}\right\}\right] .
$$

We can see that, because of the imaginary part in Eq. (20), $\Gamma$ will not be exactly -1 . Therefore, the attenuation peak will be greatly reduced due to the presence of the diffraction from the discontinuity. Moreover, at resonance, $Q_{2} \approx-1$ and $Q_{1}$ $=1$ if the other side of the discontinuity is a hard ground. Then, $\Gamma$ is approximately $\sqrt{(2 / \pi)} x(1+i)$, which approaches zero rather than -1 when $x$ is small. Hence, large cancellation will not occur at resonance. The attenuation peak will be shifted away significantly from the resonant frequency. To see whether the shift is to a lower or higher frequency, we can examine the behavior of the $Q s$ to find the condition under which $\Gamma$ may regain a value of -1 . When $x \ll 1$ and ignoring the imaginary part, Eq. (20), with $Q_{1}=1$ being a hard surface, becomes

$$
\Gamma \approx \frac{Q_{2}+1}{2} \text {. }
$$

For $\Gamma$ to approach -1 for maximum attenuation, $Q_{2}$ needs to have a largely real and negative value close to -3 .

Close to resonance, the admittance is large. For a large admittance $\beta=a+i b$, with $a$ small and negligible, the numerical distance $z$ is approximately

$$
z \approx \frac{-(1-i)}{2} \sqrt{k r_{2}} b, \text { and } z^{2}=-k r_{2}(a+\cos \theta) b-i \frac{k r_{2} b^{2}}{2} .
$$

Note that we have retained the small $(a+\cos \theta)$ term in the expression for $z^{2}$ to show the decay factor in the exponential term in $F(z)$. If $b$ is positive and large (just above a resonance), then the Heaviside factor in Eq. (7) is zero and the boundary factor $F(z)$ is again small,

$$
F(z) \approx-\frac{1}{2 z^{2}} \approx \frac{1}{k r_{2}\left(2(a+\cos \theta) b+i b^{2}\right)} \approx \frac{-i}{k r_{2} b^{2}} .
$$

In this case the real value of $Q_{2}$ above resonance does not change much from the value of -1 given by $R_{p}$. Hence, the attenuation peak frequency is not shifted towards higher frequencies. Indeed, the increasing value of the imaginary part in Eq. (23) as $b$ becomes smaller (moving away from resonance) means that the attenuation at frequencies just above resonance should decrease.

At frequencies just below resonance, $b$ is negative and large. The Heaviside function is 1 and $F(z)$ has an additional term,

$$
F(z) \approx 2 i \sqrt{\pi} z e^{-z^{2}}-i \frac{1}{k r_{2} b^{2}} .
$$

At resonance $b$ is very large and the real part of $z^{2}$ in Eq. (22) gives rise to a large decay factor in $e^{-z^{2}} \cdot F(z)$ is again very small, providing continuity in $F(z)$ between Eqs. (23) and (24) when $b$ changes from large negative values to large positive values. However, when $b$ becomes smaller [but still $>1$ and $(a+\cos \theta)]$ moving away from resonance to lower frequencies, $z^{2}$ will be dominated by the imaginary term and $e^{-z^{2}}$ becomes a largely oscillating phase factor. In this case the first term in Eq. (24) for $F(z)$ can produce reasonably large, negative real values for $Q_{2}$ to come close to -3 , and hence for $\Gamma$ to approach -1 for maximum cancellation and attenuation. Therefore, it is likely that the attenuation peak will shift to lower frequencies in the presence of a discontinuity.

Although the above analysis is based on just one discontinuity, it is expected that the general behavior should be similar when there are multiple discontinuities. However, the specific values of the attenuation will change according to specific configurations. This will be confirmed in the following sections with simulations on a variety of surfaces embedded with wells.

\section{B. Propagation over a rigid surface embedded with one well}

As discussed above, it is expected that the excess attenuation over welled surfaces will have notable changes around their resonant frequencies. This is investigated by using the nMID model to calculate the excess attenuation of a ground embedded with narrow wells. One case with a single well embedded in a rigid ground is used as examples in Fig. 9. The well has a width of $0.12 \mathrm{~m}$ and the well depth is $0.245 \mathrm{~m}$. The excess attenuation over this surface, with source and receiver heights at, respectively, 0.001 and $0.35 \mathrm{~m}$, is shown in Fig. 9. As before, the horizontal source to receiver distance is $1 \mathrm{~m}$ and the well is placed with its width in the source to receiver direction and placed midway between the source and receiver. The very small source height used here is to simulate the propagation of sound over the top surface of a T-shaped barrier where the source incidence may be considered as mostly parallel to the top surface. The small source height means that the ground reflection point is mostly on the hard portion of the ground at the 


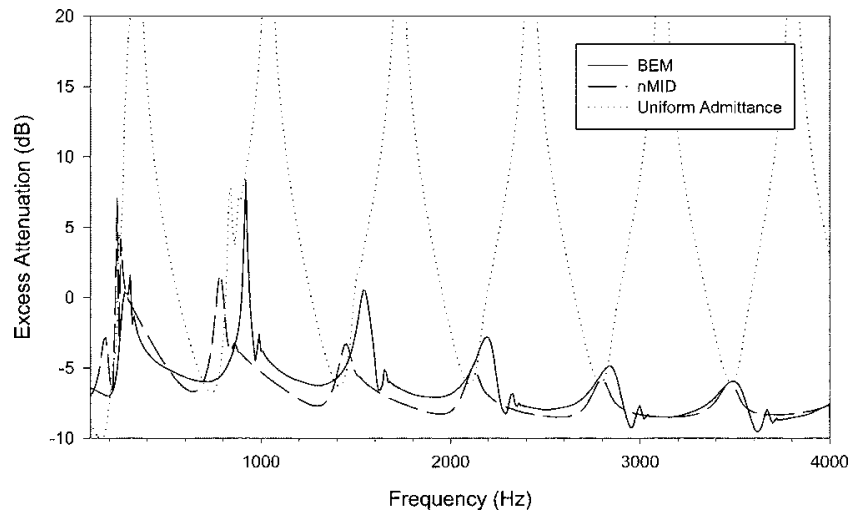

FIG. 9. Excess attenuation of propagation over a 0.12-m-wide well embedded in a rigid ground located midway between source and receiver that are $1 \mathrm{~m}$ apart. The well depth is $0.245 \mathrm{~m}$. Source and receiver heights are 0.001 and $0.35 \mathrm{~m}$, respectively.

source side before the start of the well. Figure 9 shows that the nMID model has reasonable agreement with the reference BEM calculation, and predicted the main features, i.e., the peaks in the excess attenuation just before the resonant frequencies of the wells. The discrepancies seen around the peaks are similar to those found in the cases with absorbent strips (Figs. 7 and 8) due to the closeness of the discontinuities and the near-grazing propagation. However, in the welled case the peaks are much sharper due to resonances and small losses, and the discrepancies are more apparent. The discrepancies also appear to be larger at lower frequencies. Since $k s_{j}>5$ at frequencies above approximately $300 \mathrm{~Hz}$, the assumption inherent to the hard half-plane diffraction formulation used in the model should be satisfied at frequencies above $300 \mathrm{~Hz}$. The larger discrepancies at low frequencies but above $300 \mathrm{~Hz}$ are more likely to be caused by the empirical correction for the finite impedances in the De Jong model and the interactions between the discontinuities that are ignored in the nMID model.

The result in Fig. 9 shows that the excess attenuation is indeed highly correlated with the resonances, at which the imaginary part of the admittance crosses over from a large negative value to a large positive value. The attenuation peaks just before each resonant frequency, where $\operatorname{Im}(\beta)$ is large and negative, then drops quickly to small values (negative attenuation) when $\operatorname{Im}(\beta)$ becomes large and positive. This correlates very well with our predictions from Eq. (7) on the dependence of the boundary factor $F(z)$ on the imaginary part of the admittance. Indeed, this can be seen clearly by comparing the results with that calculated for a hypothetical surface that has a uniform admittance equals to that of the well. Using this hypothetical surface, the attenuation, also shown in Fig. 9, shows strong attenuation peaks at well resonances. The peaks are much stronger than those of the BEM and nMID calculations that also account for the effect of the surrounding hard ground and the diffraction from the discontinuities. This confirms that the imaginary part of the admittance pays a crucial part in the attenuation of sound propagation over a welled surface, but the effect of the impedance discontinuities must be correctly accounted for also. The

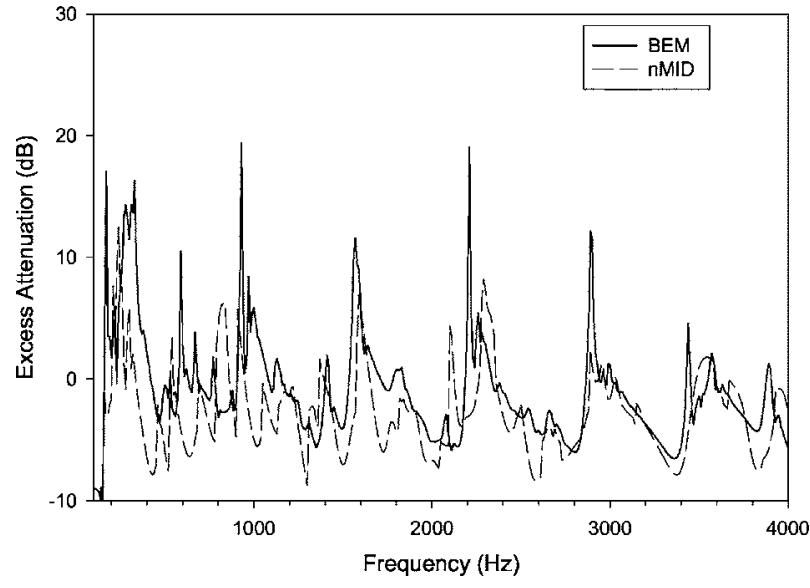

FIG. 10. Excess attenuation of propagation over seven 0.12-m-wide wells embedded in a rigid ground located midway between source and receiver that are $1 \mathrm{~m}$ apart. The well depths are $0.25,0.4,0.35,0.3,0.45,0.2$, and $0.18 \mathrm{~m}$. Source and receiver heights are 0.001 and $0.35 \mathrm{~m}$, respectively.

nMID model thus proves to be useful in providing some insight into the main factors affecting the attenuation.

\section{Propagation over multiple embedded wells}

In order to see the effect on a multiwelled surface the investigation was extended to a case with seven wells. The wells all have the same width of $0.12 \mathrm{~m}$ but the well depths are all different. The well depths from source to receiver are arbitrarily chosen to be $0.25,0.4,0.35,0.3,0.45,0.2$, and $0.18 \mathrm{~m}$. The source and receiver separation and their heights are identical to the single-well case. The result of this calculation is shown in Fig. 10. With more well depths there are more resonances within the frequency range. There are corresponding increases in the number of attenuation peaks in the graph. Thus, the general relationship between attenuation and well resonances is maintained even in the case of multiple wells. However, the magnitude and extent of these attenuation peaks do vary. Some resonances do not produce noticeable attenuation peaks at all. This is possibly caused by the diffraction effect of multiple impedance discontinuities that modifies the reflected wave. The trend of the attenuation peaks is reasonably predicted by the nMID in comparison with the BEM prediction. The nMID seems capable of accounting for the diffraction from multiple impedance discontinuities. There are however discrepancies in the positions and absolute values of the peaks between the two methods, similar to that observed earlier in Fig. 9, especially at low frequencies. Considering the closeness of the discontinuities (less than a wavelength at frequencies below $2.8 \mathrm{kHz}$ ) and the closeness of the source to the ground (source height is $0.001 \mathrm{~m}$ ), both of which are limitations of the nMID model, the nMID approximation is reasonably accurate in predicting the trend of the attenuation changes and the influences of the well resonances. Indeed, the accuracy of the nMID model improves with frequency as the width of the wells becomes comparable and eventually larger than the wavelength. The agreement between nMID and BEM is noticeably better at frequencies above $2 \mathrm{kHz}$. 
Our next test surface is a rigid surface embedded with wells that corresponds to a quadratic residue diffuser (QRD) design. The reason for choosing this surface is that previous work ${ }^{4}$ has shown that the insertion loss of a T-shaped barrier can be significantly improved by placing such a structure on the top of the T-shape. It is therefore of interest to see if this improvement in barrier performance is due to extra attenuation of sound when it propagates over the QRD covered top surface. The nMID model should be able to provide some insight into the factors that create this extra attenuation if it can correctly predict the attenuation. Again, the BEM is used as a reference in this investigation.

A QRD surface is created by a sequence of wells that have depths that are determined from a quadratic residue sequence according to the formula

$$
d_{i}=\frac{c\left(i^{2} \bmod N\right)}{N\left(2 f_{r}\right)}
$$

where $d_{i}$ is the depth of the $i$ th well, $N$ is the length of the quadratic sequence, and $f_{r}$ is the design frequency of the diffuser. Note that the design frequency is not the same as the first resonant frequency of the well with the maximum depth, which occurs at $f_{o}=c /\left(4^{*} d_{\max }\right)$, where $d_{\max }$ is the maximum depth. Full details on QRD design can be found in the literature. ${ }^{24}$

The QRD surface used in this simulation has an $N=7$ design. The design frequency is $400 \mathrm{~Hz}$. Due to the numbering sqeuence, there are only three distinct well depths and one zero depth (rigid surface). The well widths are fixed at $0.12 \mathrm{~m}$ as before. The results are shown in Fig. 11 for two source heights. The propagation geometry in Fig. 11(a) is the same as that of Fig. 10, while the source height is raised to $0.1 \mathrm{~m}$ in Fig. 11(b). Because of the smaller number of distinct well depths there are less distinct resonant frequencies, and Fig. 11 shows correspondingly less attenuation peaks than Fig. 10, which has seven distinct wells. Note that once again the nMID model provides reasonable prediction over the trend of the attenuation despite noticeable errors in the peak magnitudes. As the source height is raised in Fig. 11(b), the accuracy of nMID improves, as is expected from the assumption of the nMID model.

When the source height is very close to the surface $(0.001 \mathrm{~m})$, which we used to approximate the incidence condition over the top surface of a T-shaped noise barrier, the results in Figs. 10 and 11 show that the nMID predictions have tolerable agreement with the BEM predictions. There are still noticeable discrepancies in the values of the peak frequencies and magnitudes at low frequencies. However, the general trends of the attenuation, and in particular the influence of the well resonances and the effect of multiple diffractions, between the two predictions are in good agreement. We believe that this shows that the nMID is sufficiently accurate to explain the effect of a QRD surface on the top of a T-shaped barrier, although it may not be accurate enough to be used as a means to predict the absolute magnitude of the barrier performance. (a) Source height $0.001 \mathrm{~m}$

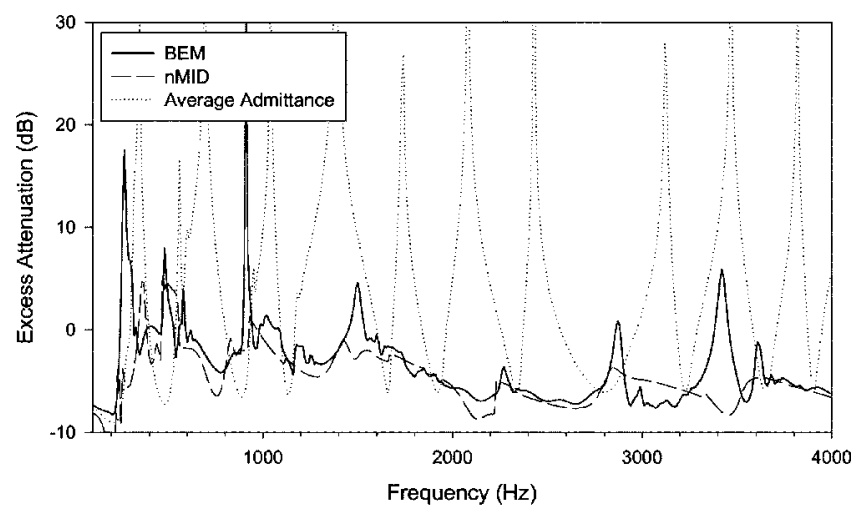

(b) Source height $0.1 \mathrm{~m}$

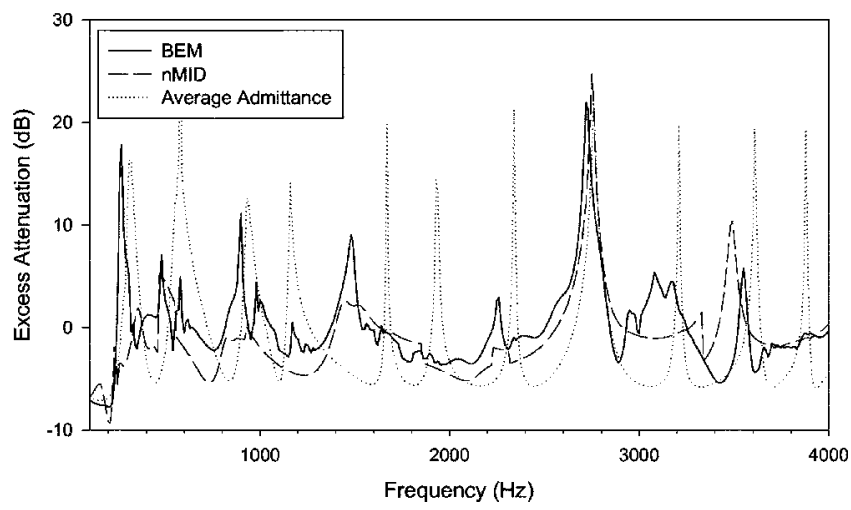

FIG. 11. Excess attenuation of propagation over $N=7$ QRD surfaces embedded in a rigid ground located midway between source and receiver that are $1 \mathrm{~m}$ apart. The well widths are fixed at $0.12 \mathrm{~m}$. Source and receiver heights are 0.001 and $0.35 \mathrm{~m}$, respectively.

\section{Average admittance effect}

Nyberg ${ }^{16}$ demonstrated that the Helmholtz equation with the boundary condition for a point source above an infinite plane surface with periodic impedance strips can be solved by using a Fourier transform approach. The wave propagation over a two-valued, infinitely periodic striped impedance can be approximated by employing the area-averaged admittance in the calculation of the reflection coefficient,

$$
\beta_{\mathrm{ave}}=\frac{\left(w_{1} \beta_{1}+w_{2} \beta_{2}\right)}{\left(w_{1}+w_{2}\right)},
$$

where $w_{1}$ and $w_{2}$ are the widths of the two types of strips, and $\beta_{1}$ and $\beta_{2}$ are the corresponding admittance values. The assumption made is that $\left(w_{1}+w_{2}\right)$ is much smaller than the acoustic wavelength. In our case the width of each well (strip) is smaller than a wavelength. However, since the strips are not periodic, the total width of all the nonperiodic strips added together is larger than or comparable to a wavelength in most of the frequency range. Hence, Nyberg's approximation cannot be applied directly here. In earlier sections we have seen that the admittance of the well plays a deterministic role in the attenuation of sound propagation over a surface with a single well. It is therefore of interest to see if the area-averaged admittance over multiple wells also 
correlates with the attenuation phenomenon. At resonance the average admittance is dominated by that of the well in resonance and therefore the attenuation should be correlated with the average admittance. In Fig. 11 the prediction using the averaged admittance without the diffraction terms from the discontinuities, i.e., on a hypothetical surface of uniform admittance equals to the average admittance, is also shown. Without accounting for the diffraction, the prediction shows a strong attenuation peak at every resonant frequency. Although some of these peaks do coincide with the attenuation peaks predicted by the BEM and nMID models (especially at frequencies below $1 \mathrm{kHz}$ when the acoustic wavelength is longer than the overall width of all the wells), fewer peaks appear in the more accurate predictions. This again shows the importance of accounting for the diffraction from the impedance discontinuities, and suggests that the effect of the discontinuities actually reduces the attenuation peaks both in number and in magnitude. However, there is also evidence, most noticeably at frequencies below $1 \mathrm{kHz}$ in Fig. 11, that the diffraction terms also broaden the peaks slightly and also do not allow the attenuation to drop to large negative values immediately after each resonance. It therefore appears that the imaginary part of the areaaveraged admittance can be used as a pointer for optimizing the attenuation provided by such surfaces, provided that the effect of the diffraction from the discontinuities is accounted for properly, by either the BEM or nMID to obtain the correct attenuation levels.

\section{CONCLUSIONS}

The original De Jong model for the calculation of diffraction from an impedance discontinuity has an inherent assumption of transition from a hard to a softer impedance. Unfortunately this assumption has been overlooked in many of the subsequent works ${ }^{13,14}$ that attempted to extend the model to cases with multiple impedance discontinuities. This paper has shown that such extensions are invalid because of this assumption of hard to soft transition. In one previous work ${ }^{13}$ where the extension to one impedance strip (two impedance discontinuities) did work, there was no clear explanation as to the adjustment in the equations that were used. Consequently, some confusion was found in later work which considered the extension to be inconsistent and should be corrected. In this paper we used the acoustic reciprocity condition to derive a modified form of the De Jong equation that does not have the restriction of hard to soft impedance transition. This modification clarifies the previously unexplained adjustment made in the equations of Hothersall et $a l .{ }^{13}$ This modified equation was then used to obtain a new multiple impedance discontinuities model, the nMID. It was shown that this nMID model agrees well with the accurate boundary element method when applied to single and multiple impedance strips that are made up of typical porous surfaces. As expected, due to the inherent assumption of the De Jong approximation and the disregard of intercoupling effects between discontinuities, the accuracy decreases as the source or receiver height decreases (nearer grazing incidence) and when the width of the strips or the distance be- tween the discontinuities becomes shorter than the acoustic wavelength. Nevertheless, the simulations presented in this paper have shown that the nMID is still capable of predicting the main features of the excess attenuation spectrum under these unfavorable conditions.

One of the main objectives of this work is to investigate the attenuation of sound propagation over multiple impedance strips that are created by rigid wells of different depths. The admittance of rigid wells has a different behavior from that of porous surfaces. The extreme values created by well resonances mean that the admittance value can be close to the limits of the valid range of useful approximations that are used to calculate the spherical reflection coefficient. It has been shown, by examining the behavior of the spherical reflections coefficient at different values of admittance, that the wells can have large effect on the sound propagation when the imaginary part of the admittance is large and negative (with a $e^{-i \omega t}$ convention), which occurs just before a well resonance. This correlation was confirmed by simulations on surfaces with single and multiple wells. It was found that the attenuation peaks at frequencies just below the resonant frequencies, at which the imaginary part of the admittance becomes very large and negative. In case of multiple wells, the area-average admittance gives a rough indication to this behavior and can be considered as a first approximation pointer for quick optimization of the attenuation over such surfaces. However, diffraction from the impedance discontinuities modifies and substantially reduces the magnitudes of the attenuation peaks, and therefore must be properly accounted for. Overall, it was found that the nMID model is capable of accounting for these effects, although discrepancies are more noticeable at the sharp attenuation peaks at lower frequencies.

${ }^{1}$ L. A. M. van Der Heijden and M. J. M. Martens, "Traffic noise reduction by means of surface wave exclusion above parallel grooves in the roadside," Appl. Acoust. 15, 329-339 (1982).

${ }^{2}$ P. F. van Tol and H. A. Holties, "A reciprocal method to evaluate low, close to track noise barriers," J. Acoust. Soc. Am. 105(2), 949 (1999).

${ }^{3}$ K. Fujiwara, D. C. Hothersall, and C. H. Kim, "Noise barriers with reactive surfaces," Appl. Acoust. 53(4), 225-272 (1998).

${ }^{4}$ M. R. Monazzam and Y. W. Lam, "Performance of profile single noise barriers covered with quadratic residue diffusers," Appl. Acoust. 66, 709730 (2005)

${ }^{5}$ W. Zhu, M. R. Stinson, and G. A. Daigle, "Scattering from impedance gratings and surface wave formation," J. Acoust. Soc. Am. 111(5), 19962012 (2002)

${ }^{6}$ B. O. Enflo and P. H. Enflo, "Sound wave propagation from a point source over a homogeneous surface and over a surface with an impedance discontinuity," J. Acoust. Soc. Am. 82, 2123-2135 (1987).

${ }^{7} \mathrm{~K}$. B. Rasmussen, "A note on the calculation of sound propagation over impedance jumps and screens," J. Sound Vib. 84, 598-602 (1982).

${ }^{8}$ K. E. Gilbert and M. J. White, "Application of the parabolic equation to sound propagation in a refracting atmosphere," J. Acoust. Soc. Am. 85, 630-637 (1989).

${ }^{9}$ S. N. Chandler-Wilde and D. C. Hothersall, "Sound propagation above an inhomogeneous impedance plane," J. Sound Vib. 98, 475-491 (1985),

${ }^{10}$ B. A. De Jong, A. Moerkerken, and J. D. Van Der Toorn, "Propagation of sound over grassland and over an earth barrier," J. Sound Vib. 86(1), 23-46 (1983).

${ }^{11}$ P. Koers, "Diffraction by an absorbing barrier or by an impedance transition," Proceedings of Internoise'83, 311-314 (1983).

${ }^{12}$ G. A. Daigle, J. Nicolas, and J.-L. Berry, "Propagation of noise above ground having an impedance discontinuity," J. Acoust. Soc. Am. 77(1), 127-138 (1985). 
${ }^{13}$ D. C. Hothersall and J. N. B. Harriott, "Approximate models for sound propagation above multi-impedance plane boundaries," J. Acoust. Soc. Am. 97(2), 918-926 (1995).

${ }^{14} \mathrm{P}$. Boulanger, T. Waters-Fuller, K. Attenborough, and K. M. Li, "Models and measurements of sound propagation from a point source over mixed impedance ground," J. Acoust. Soc. Am. 102(3), 1432-1442 (1997).

${ }^{15}$ M. R. Bassiouni, C. R. Minassian, and B. Chang, "Prediction and experimental verification of far-field sound propagation over varying ground surface," Proceedings of Internoise' 83, 287-290 (1983).

${ }^{16} \mathrm{C}$. Nyberg, "The sound field from a point source above a striped impedance boundary," Acta Acust. (Beijing) 3, 315-322 (1995).

${ }^{17}$ C. F. Chien and W. W. Soroka, "Sound propagation along an impedance plane," J. Sound Vib. 43(1), 9-20 (1975).

${ }^{18}$ M. E. Delany and E. N. Bazely, "Acoustical properties of fibrous absorbent materials" Appl. Acoust. 3, 105-116 (1970).

${ }^{19}$ T. Wu, T. J. Cox, and Y. W. Lam, "From a profiled diffuser to an optimized absorber," J. Acoust. Soc. Am. 108(2), 634-650 (2000).

${ }^{20}$ R. B. Lawhead and I. Rudnick, "Acoustic wave propagation along a constant normal impedance boundary," J. Acoust. Soc. Am. 23, 546-549 (1951).

${ }^{21}$ L. E. Vogler, "A note on the attenuation function for propagation over a flat layered earth," IEEE Trans. Antennas Propag. AP-12, 240-242 (1964).

${ }^{22}$ R. J. King and G. A. Schalk, "Ground wave attenuation function for propagation over a highly inductive earth," Radio Sci. 2 (New Series), 687-693 (1967).

${ }^{23} \mathrm{M}$. R. Stinson, "A note to the use of an approximate formula to predict sound field above an impedance plane due to a point source," J. Acoust. Soc. Am. 98, 1810-1812 (1995).

${ }^{24}$ M. R. Schroeder, "Binaural dissimilarity and optimum ceilings for concert halls: More lateral sound diffusion," J. Acoust. Soc. Am. 65, 958-963 (1979). 